Pacific Journal of Mathematics

SUPPLEMENTED MODULES OVER DEDEKIND DOMAINS 


\title{
SUPPLEMENTED MODULES OVER DEDEKIND DOMAINS
}

\author{
JUTTA HAUSEN
}

The $R$-module $M$ is said to be supplemented if every submodule of $M$ has a minimal supplement. For $R$ a Dedekind domain, we relate this lattice theoretical condition to direct decompositions of $M$, the smallness of the radical $J(M)$ of $M$, the semi-simplicity and lifting of decompositions of $M / J(M)$, and the existence of quasi-projective covers. If $M$ is contained in some $R$-module as a small submodule, $M$ is said to be a small module. The structure of all supplemented and all small $R$-modules is determined and it is shown that, for $R$ local, the smallness of $J(M)$, the smallness of $M$, and $M$ being a supplemented reduced module are equivalent conditions.

1. Introduction. $H$. Bass calls a ring $R$ semi-perfect if every epimorphic image of the $R$-module $R$ has a projective cover. In [2] he proved that $R$ is semiperfect if and only if $R / J(R)$ is semisimple and decompositions of $R / J(R)$ can be lifted to $R$. Erika Mares generalized this concept to modules [15]. Independently, the same class of modules was considered by Y. Miyashita [16]. Combining results of $[12 ; 15 ; 16]$ we obtain the following theorem. Here, an $R$-module $M$ is called supplemented if, given any submodule $N$ of $M$ there exists a submodule $S$ of $M$ which is minimal with respect to the condition that $M=N+S$ [7]. Throughout, $J(M)$ denotes the Jacobson radical of $M$.

THEOREM $[12 ; 15 ; 16]$. The following properties of the projective module $M$ are equivalent.

(1) Every epimorphic image has a projective cover.

(2) $M / J(M)$ is semi-simple, direct decompositions of $M / J(M)$ can be lifted to $M$, and $J(M)$ is small in $M$.

(3) $M$ is a direct sum of sum-irreducible modules and $J(M)$ is small in $M$.

(4) $M$ is supplemented.

(5) $M$ is amply supplemented.

If, in addition, $M$ is a progenerator, then (1)-(5) are equivalent to

(6) Every M-generated module has a projective cover.

Various parts of this result have been generalized by J. S. Golan [7], G. Hauger [8], and R. Wisbauer [20]. In particular, Golan has shown that, for $M$ quasi-projective with small radical, (5) implies 
(7) Every non-small submodule of $M$ contains a non-zero direct summand of $M[7 ;$ p. 178, 3.3].

In all these generalizations, however, some type of projectivity condition was imposed upon $M$. The purpose of this article is to drop this restriction and determine how properties (1)-(7) relate to each other for arbitrary $R$-modules $M$.

In order to compensate for not requiring $M$ to be projective, we let $R$ be a Dedekind domain. Attention will be focused on the supplemented $R$-modules. After establishing their structure (Theorem 4.14) we prove that, for $M$ reduced, properties (3), (4), and (5) remain equivalent; the other properties need to be modified somewhat, changing from projective covers to quasi-projective covers and from direct decompositions to weak direct decompositions (Theorem 4.17). If $M$ is not reduced, (4) is still equivalent to weakened versions (2) and (3) (Theorem 4.20), but (4) and (5) cease to be equivalent, thus providing a negative answer to the question whether supplemented modules need to be amply supplemented.

The material is organized as follows. After a preliminary $\S 2$, we consider the category of small $R$-modules in the sense of Leonard [14]. In $\S 3$, the structure of small $R$-modules is determined (Theorem 3.6). We show that in some instances the smallness of a module is equivalent to the smallness of its radical (Proposition 3.7). The results on small modules are applied to questions concerning the existence of quasi-projective covers. Section 4 is devoted to supplemented modules. We prove that, for $R$ not a field, the $R$-module $M$ is supplemented if and only if: (i) for each maximal ideal $P$ of $R$, the set $M_{P}$ of all elements of $M$ which are annihilated by a power of $P$ is a direct sum of a bounded and an artinian submodule; and (ii) $M=\bigoplus_{P} M_{P}$ where the summation ranges over all prime ideals $P$ of $R$, and $M_{0} \neq 0$ implies $R$ is local and $M_{0}$ is a direct sum of finitely many torsion-free modules of rank one (Theorem 4.14). As a consequence we obtain the fact that the class of supplemented modules need not be closed with respect to submodules, nor need it be closed with respect to extensions. We then consider the relationship between $M$ being supplemented and the other conditions (1)-(7) listed above.

It is not surprising that the strongest analogy to the projective case occurs under the assumption that $M$ is reduced. After all, for $R$ a Dedekind domain, a module $M$ over $R$ is reduced if and only if, for every submodule $N$ of $M, N=J(N)$ implies $N=0$. This corresponds to Axiom 5 in Eilenberg's definition of a "Perfect Category" [3; p. 331f]. In essence, a category of modules is "perfect" if every module in it has a projective cover. It was this terminology that later motivated $\mathrm{H}$. Bass' nomenclature of perfect and semiper- 
fect rings [2; p. 467].

2. Preliminaries. Throughout, $R$ will be a commutative ring with identity. All modules are unital left $R$-modules. Notation and terminology will follow [1] unless explained otherwise. If $M$ is a module, $S \leqq M$ signifies that $S$ is a submodule of $M$. The Jacobson radical of $M$ will be denoted by $J(M)$. For $I$ an ideal in $R$, we let

$$
I M=\{r a \mid r \in I, a \in M\},
$$

and abbreviate $I\{x\}$ to $I x$ if $x \in M$. If, for $r \in R, r x=0$ implies $r=0$, then $x$ is a non-torsion element; otherwise, $x$ is said to be a torsion element. A submodule $S$ of $M$ is small in $M$, in symbol

$$
S \ll M,
$$

if $T \leqq M$ and $M=S+T$ imply $T=M$. Frequent use will be made of the fact that homomorphisms map small submodules to small submodules [1; p. 74, 5.18], and that $S^{\prime} \leqq S \ll M$ implies $S^{\prime} \ll M$. Also, $J(M)$ is the sum of all small submodules of $M[1$, p. 120, $9.13]$.

If $C_{i} \leqq M, i=1,2$, such that

$$
M=C_{1}+C_{2} \text { and } C_{1} \cap C_{2} \ll C_{i}, i=1,2,
$$

we say that $M$ is the weak direct sum of $C_{1}$ and $C_{2}$, and write

$$
M=C_{1} \bigoplus_{\|} C_{2}
$$

to denote this fact. The $C_{2}$ are called weak direct summands of $M$. It will follow from 4.1 that $M=C_{1} \bigoplus_{W} C_{2}$ if and only if $C_{1}$ and $C_{2}$ are minimal supplements of each other: a minimal supplement (in $M$ ) of $N \leqq M$ is a submodule $S$ of $M$ such that (i) $M=N+S$, and (ii) $M \neq N+S^{\prime}$ for all $S^{\prime} \leqq S$. If every submodule of $M$ has a minimal supplement in $M$, then $M$ is said to be supplemented; $M$ is amply supplemented if, given submodules $N$ and $N^{\prime}$ of $M$ with $M=N+N^{\prime}$, there exists a minimal supplement $S$ of $N$ in $M$ such that $S \leqq N^{\prime}$. Thus, $M$ is amply supplemented if and only if $M=$ $N+N^{\prime}$ implies $M=C \bigoplus_{W} C^{\prime}$ for some $C \leqq N, C^{\prime} \leqq N^{\prime}$.

For the most part, $R$ will be a Dedekind domain. $R$ is said to be proper if $R$ is not a field. Throughout, $K$ will denote the quotient field of $R$, and $\boldsymbol{P}$ is the set of all non-zero prime ideals of $R$. The relevant facts on modules over Dedekind domains can be found in Kaplansky's paper [9]. We summarize: for each $\boldsymbol{P} \in \boldsymbol{P}$, let $M_{P}$ denote the set of all $x \in M$ such that $P^{n} x=0$ for some integer $n \geqq 0$, and let 


$$
R_{P}=\left\{r s^{-1} \in K \mid r, s \in R, s \notin P\right\}
$$

be the localization of $R$ at $P$. Then $M_{P}$ is a submodule of $M$, and $M_{P}$ can be regarded as a module over $R_{P}$. Since $R_{P}$ is a discrete valuation ring, hence, a principal ideal domain, the theory of Abelian groups as developed in [10] applies [10; p. 36]. If $T$ denotes the torsion submodule of $M$, then

$$
T=\bigoplus_{P \in P} M_{P},
$$

and $M / T$ is torsion-free. Every torsion free $R$-module $N \neq 0$ contains submodules $F$ and $S$ such that $F$ is free, $N / F$ is torsion, and $N / S$ is isomorphic to a non-zero submodule of the $R$-module $K$. Since $K / R$ is torsion,

$$
K / R=\bigoplus_{P \in \mathbf{P}}(K / R)_{P}
$$

For $P \in P,(K / R)_{P}$ is called the module of type $P^{\infty}$; we will denote it by $R\left(P^{\infty}\right)$. Every divisible $R$-module is a direct sum of copies of $K$ and $R\left(P^{\infty}\right)$ for various $P \in \boldsymbol{P}$. Divisible submodules are direct summands. Every $R$-module $M$ contains a maximal divisible submodule, which we shall denote by $d M$. If $d M=0$ then $M$ is said to be reduced. A module $B$ is called bounded if $I B=0$ for some nonzero ideal $I$ of $R$. Since nonzero ideals are finite products of maximal ideals, this definition is equivalent to $r B=0$ for some $0 \neq r \in R$. If $B$ is a bounded submodule of $M$ and $M / B$ is torsionfree then $B$ is a direct summand of $M$. Bounded modules are direct sums of cyclics.

Note that, if $R$ is a proper Dedekind domain, $J(M)=\bigcap_{P \in P} P M$; also, $R$ is Noetherian.

Lemma 2.1. For a module $M$ over the proper Dedekind domain $R$, the following conditions are equivalent.

(i) $M$ is injective;

(ii) $J(M)=M$;

(iii) $M$ is divisible;

(iv) $M$ is a direct sum of copies of $K$ and $R\left(P^{\infty}\right)$, for various $P \in P$.

Proof. By [17; p. 196, 6.1], (i) and (ii) are equivalent; the equivalence of (i) and (iii) is well known [19; p. 33, 2.6, and p. 108, 4.25]. The proof is completed using [9; p. 335, Theorem 7] and [19; p. 82, 4.1].

We will need the following rather technical result. 
Lemma 2.2. Let $M$ be a module over the Dedekind domain $R$, let $I$ be a nonzero proper ideal of $R$ and let $x \in M$ such that $I x$ is a weak direct summand of $M$. Then $R x$ is torsion,

Proof. If $M=I x \bigoplus_{w} T$ then $R x=I x+(R x \cap T)$ and $I x \cap(R x \cap$ $T) \ll I x$. Assume $R x$ is not torsion. Then $R x \cong R$ and

$$
R=I+J, I \cap J \ll I
$$

for some ideal $J$ of $R$. By hypothesis, $I \leqq P$ for some $P \in P$. From (2.3) and [13; p. 53. Exercise 2(b)] we obtain

$$
I J=I \cap J \leqq J(I) \leqq P I .
$$

By [13; p. 137, 6.20 (8)], there exists an ideal $J^{\prime}$ in $R$ such that

$$
I J=P I J^{\prime} \text {. }
$$

But then $J=P J^{\prime} \leqq P[13 ;$ p. $137,6.20(4)]$ so that $P$ contains both $I$ and $J$. By (2.3), this is not possible. The lemma is proven.

The module $M$ is called sum-irreducible if, for any pair $A$ and $B$ of proper submodules of $M, A+B$ is a proper submodule [16; $\mathrm{p}$. 97]. A module whose lattice of submodules is a chain certainly is sum-irreducible. Thus, for each $P \in P$, the modules $R\left(P^{\infty}\right)$ and $R / P^{n}$, $n$ a positive integer, are sum-irredducible and, if $R$ is local, so are $R$ and $K$ and hence all torsion-free $R$-modules of rank one [10; $\mathrm{p}$. 45]. Clearly, factor modules of sum-irreducible modules are sumirreducible. Because of

$$
K / R=\bigoplus_{P \in P} R\left(P^{\infty}\right),
$$

$K$ therefore is sum-irreducible only if $R$ is local. Miyashita has shown that a module $M$ with $J(M) \neq M$ is sum-irreducible if and only if $M / J(M)$ is a simple module; in particular, such a module is cyclic [16; p. 97, 3.5]. Thus, if $R$ is not local, the only sum-irreducible $R$-modules are the submodules of $R\left(P^{\infty}\right), P \in P$.

We collect this information. By definition, $P^{0}=R$.

Lemma 2.4. Let $M$ be a module over the Dedekind domain $R$. Then $M$ is sum-irreducible if and only if one of the following conditions is met:

(i) $M \cong R / P^{n}, P \in P, n \geqq 0$ an integer;

(ii) $M \cong R\left(P^{\infty}\right), P \in P$;

(iii) $R$ is local and $M$ is torsion-free of rank one.

3. Small modules and quasi-projective covers. A small $R$ module is any $R$-module $S$ such that $S \ll M$ for some $R$-module $M$ 
[14].

(3.1) The class of small $R$-modules is closed with respect to submodules, finite direct sums, and epimorphic images $[17 ;$ p. 189, $3.2]$.

W. W. Leonard has shown that the $R$-module $S$ is small if and only if $S$ is a small submodule of its injective envelope $[14 ;$ p. 527, Theorem 1]. Thus:

(3.2) The $R$-module $S$ is small if and only if it is a small submodule in every injective module containing $S$ [17; p. 190, 4.2].

In order to determine the small modules over Dedekind domains, we will need the following result.

Proposition 3.3. If $R$ is a hereditary ring, then the $R$-module $S$ is small if and only if $T \leqq S$ and $S / T$ injective imply $S=T$.

Proof. Let $E$ be an injective envelope of $S[1 ;$ p. 207, 18.10], $S \leqq E$. Assume $S$ is small and let $T \leqq S$ such that $S / T$ is injective. Then $S / T$ is a direct summand of $E / T[1 ;$ p. 206, 18.7] proving $S / T=0$, by 3.1 and 3.2. For the converse, let $C \leqq E$ such that $E=S+C$. Then $E /(S \cap C)=S /(S \cap C) \oplus C /(S \cap C)$ and, since $R$ is hereditary, $S /(S \cap C) \cong E / C$ is injective. By hypothesis, $S=S \cap$ $C$, hence $E=C$, completing the proof.

We obtain the following consequence using Lemma 2.1 and [4; p. 30, 18.3].

CoRollary 3.4. Let $M$ be a module over a proper Dedekind domain. Then the following conditions are equivalent.

(i) $M$ is small.

(ii) If $N \leqq M$ such that $J(M / N)=M / N$ then $N=M$.

(iii) For all $P \in P, R\left(P^{\infty}\right)$ is not an epimorphic image of $M$.

(iv) Every proper submodule of $M$ is contined in a maximal submodule.

By [4; p. 30f, 18.3(6)], the equivalent properties (ii) and (iv) imply that $J(M)$ is small in $M$. Hence:

CoRollary 3.5. If $M$ is a small module over a Dedekind domain $R$ then $J(M) \ll M$.

Obviously, the converse of 3.5 is false: if $J(R)=0$ then $J(F)=0$ for every free module $F$ over $R[1 ;$ p. 196, 17.10] even though $F$ need not be small.

If $R$ is a field, the only small $R$-module is the zero-module. For proper Dedekind domains we have the following structure theorem. 
THEOREM 3.6. Let $M$ be a module over the proper Dedekind domain $R$. Then $M$ is small if and only if $M$ contains a free submodule $F$ of finite rank such that $M / F$ is a torsion module and, for each $P \in P,(M / F)_{P}$ is bounded.

Proof. Let $F$ be a free submodule of $M$ such that $M / F$ is torsion. Assume, firstly, that $M$ is small. Let $P$ be a nonzero prime ideal of $R$. By 3.4, $R\left(P^{\infty}\right)$ is no epimorphic image of $M$. Hence $(M / F)_{P}$ is bounded and, since $R\left(P^{\infty}\right)$ is countably generated, $F$ must have finite rank. Conversely, suppose that $M$ is of this form. Then 0 is the only divisible factor module of $M$, and the proof is completed by 2.1 and 3.4 .

Proposition 3.7. Let $M$ be a module over the Dedekind domain $R$. If either $M$ is torsion or $R$ is local, then the following conditions are equivalent.

(i) $M$ is small;

(ii) $J(M) \ll M$;

(iii) $J(M)$ is small;

(iv) $M=F \oplus \bigoplus_{P \in P} M_{P}$ where, for each $P \in \boldsymbol{P}, M_{P}$ is bounded, and $F \neq 0$ implies $R$ is local and $F$ is free of finite rank.

Proof. By Theorem 3.6, (iv) implies (i), which in turn, using 3.5, implies (ii), and (ii) implies (iii). Assume (iii). If $R$ is local with maximal ideal $P$, then $P M=J(M)$; by Theorem 3.6, $J(M)$ contains a free submodule $F$ of finite rank such that $P^{n} J(M) \cong F$ and hence $P^{n+1} M \subseteq F$ for some positive integer $n$. Since submodules of free $R$-modules are free [10; p. 44, Lemma 15], $M$ in this case is of the form (iv). If $M=\bigoplus_{P \in P} M_{P}$ is torsion then $J(M)=$ $\bigoplus_{P \in P} J\left(M_{P}\right)$. Since, for each $P \in P, P M_{P}=J\left(M_{P}\right)$ and $J\left(M_{P}\right)$ is bounded by 3.6, $M_{P}$ is bounded, completing the proof.

Let $M$ and $X$ be $R$-modules. $X$ is called a projective cover of $M$ if $X$ is projective and $M \cong X / S$ for some small submodule $S$ of $X[1 ;$ p. 199]. Though dual to the concept of injective envelopes, projective covers need not exist. For example, the only $Z$-modules which have projective covers are the free ones $[2 ;$ p. 467]. Generalizations of projective covers were considered in $[7 ; 8 ; 18 ; 20 ; 21]$. Wu and Jans call $X$ a quasi-projective cover of $M$ if $X$ is quasiprojective, if $M \cong X / S$ for some small submodule $S$ of $X$, and if $T \leqq S, X / T$ quasi-projective imply $T=0$ [21; p. 443]. We find it more natural not to require the last condition and use the terminology of [18] instead.

DEFinition 3.8. The module $X$ is a quasi-projective cover of 
$M$ if $X$ is quasi-projective and $M \cong X / S$ for some small submodule $S$ of $M$.

As J. S. Golan has shown, a module has a quasi-projective cover according to our definition if and only if it has a quasi-projective cover in the sense of $\mathrm{Wu}$ and Jans $[7 ; \mathrm{p} .176,1.7]$. Since we are concerned with the existence of quasi-projective covers only, the difference in terminology can be ignored.

We shall need two results on the existence of quasi-projective covers.

Lemma 3.9. Let $R$ be a non-local Dedekind domain and $P \in P$. Then $R\left(P^{\infty}\right)$ has no quasi-projective cover.

Proof. Suppose $X$ is quasi-projective, $S \ll X$ and $X / S \cong R\left(P^{\infty}\right)$, for some $P \in P$. We first want to show that $S$ must be torsion. Assume the contrary. Since $R$ is not local, there exists a free submodule $F$ of $S$ such that $S / F$ is torsion and

$$
S / F=(S / F)_{P} \oplus T / F, T / F \neq 0 .
$$

Because of $R\left(P^{\infty}\right) \cong X / S \cong(X / F) /(S / F)$,

$$
X / F=(X / F)_{P} \oplus T / F,(X / F)_{P} \neq X / P .
$$

Hence $X / F=(X / F)_{P}+S / F$, and $S / F$ is not small in $X / F$. This contradicts $S \ll X$ and we have shown that $S$ is torsion. Since $X / S \cong R\left(P^{\infty}\right)$, there exist decompositions

$$
S=S_{P} \oplus U, X=X_{P} \oplus U .
$$

By $3.5, S_{P}$ is bounded. From

$$
R\left(P^{\infty}\right) \cong X / S \cong X_{P} / S_{P}
$$

we deduce that $X_{P}$ cannot be reduced. Thus

$$
X=\mathrm{A} \oplus B, A \cong R\left(P^{\infty}\right) .
$$

But [6; p. 6, Lemma 4] implies that every proper submodule of $A$ is a direct summand of $X$ which is impossible. The lemma is proven.

THEOREM 3.10. If $M=\bigoplus_{P \in P} M_{P}$ is a torsion module over a Dedekind domain such that, for each $P \in P, M_{P}$ is bounded then $M$ has a quasi-projective cover $X$. Moreover, $X=\bigoplus_{P \in P} X_{P}$ where, for each $P \in P, X_{P}$ is bounded.

Proof. For $P \in P$, let $n$ be a positive integer such that $P^{n} M_{P}=$ 0 . Then $M_{P}$ is a module over the ring $R / P^{n}$ which is a perfect ring 
[1; p. 315, 28.4]. Thus, there exists a $R / P^{n}$-module $X_{P}$ which is a projective cover of $M_{P}$. Regarded as $R$-module, $X_{P}$ is quasi-projective $[1 ;$ p. 191, Exercise 17] and, thus, a quasi-projective cover of $M_{P}$. It follows from [6; p. 6, Lemma 3] that $X=\bigoplus_{P \in P} X_{P}$ is quasiprojective. Clearly, $X$ is a quasi-projective cover of $M$, and each $X_{P}$ is bounded.

4. Supplemented modules. The following results are contained in $[16 ;$ p. $87,1.3$, and p. $90,1.11]$ and will be used frequently. For the time being, $R$ may be any ring with identity.

4.1. If $N, S \leqq M$ then $S$ is a minimal supplement of $N$ if and only if

$$
M=N+S, \quad N \cap S \ll S .
$$

4.2. If $M=N+S, \quad N \cap S \ll S$, then $J(S)=S \cap J(M)$ and $J(M)=[N \cap J(M)]+[S \cap J(M)]$.

Combining these with $[16 ;$ p. $87,1.3]$ we obtain

4.3. If $M=C_{1} \oplus_{W} C_{2}$ then $J(M)=J\left(C_{1}\right)+J\left(C_{2}\right)$, and $J(M) \ll M$ implies $J\left(C_{i}\right) \ll C_{i}, i=1,2$.

LEMMA 4.4. Let $M=N+S, \quad N \cap S \ll S . \quad$ If $M / N=J(M / N)$ then $S=J(S)$.

Proof. Since $M / N$ and $S /(N \cap S)$ are isomorphic,

$$
S /(N \cap S)=J[S /(N \cap S)]=J(S) /(N \cap S),
$$

by $[1 ;$ p. $121,9.15]$. Hence $S=J(S)$.

The following simple results can be found in $[11 ;$ p. $245,11.1 .4$ (3), p. $268,4 . b)]$.

4.5. If $A \leqq M$ and $M$ is supplemented then $M / A$ is supplemented.

4.6. If $A, B \leqq M$ and $A$ and $B$ are supplemented then $A+B$ is supplemented.

Together they imply

4.7. $A \oplus B$ is supplemented if and only if both $A$ and $B$ are supplemented.

An examination of the proof of $[7 ; p .178,3.6]$ yields

4.8. If $M$ is supplemented then $M / J(M)$ is semi-simple.

If $J(M) \ll M$ and $M / J(M)$ is semi-simple, then $M$ need not be supplemented. This can be seen from the $Z$-module consisting of all rational numbers with square-free denominator. However, we have the following result. We say that weak direct decompositions of $M / J$ can be lifted to $M$ if, given $J \leqq B_{i} \leqq M, i=1,2$, such that $M / J=B_{1} / J \bigoplus_{W} B_{2} / J$, there exist $C_{i} \leqq M$ such that $M=C_{1} \bigoplus_{W} C_{2}$ and 
$C_{i}+J=B_{i}, i=1,2$. Clearly, weak direct decompositions of semisimple modules are direct decompositions.

Proposition 4.9. If $J(M) \ll M$, if $M / J(M)$ is semi-simple, and if weak direct decompositions of $M / J(M)$ can be lifted to $M$, then $M$ is supplemented.

Proof. Let $N \leqq M$ and put $J=J(M)$. Then $M / J=(N+J) /$ $J \oplus T / J$, hence $M=C_{1} \oplus_{W} C_{2}$ such that $C_{1}+J=N+J, C_{2}+J=T$, and $J\left(C_{2}\right)=J \cap C_{2} \ll C_{2}$, by 4.2 and 4.3. It follows that $M=N+$ $J+C_{2}=N+C_{2}$, and

$$
N \cap C_{2} \leqq(N+J) \cap\left(C_{2}+J\right) \cap C_{2}=(N+J) \cap T \cap C_{2}=J \cap C_{2} .
$$

Thus $N \cap C_{2} \ll C_{2}$, and 4.1 completes the proof.

From now on we assume that $R$ is a proper Dedekind domain. By 2.1, coinciding with their radical is equivalent for $R$-modules to be divisible. Thus, Lemma 4.4 and Corollary 3.4 imply:

4.10. If $S \leqq M$ is a minimal supplement of $N \leqq M$ and $M / N$ is divisible, then $S$ is divisible.

4.11. Reduced supplemented modules over Dedekind domains are small.

The following observation will be useful.

LEMma 4.12. Let $P \in \boldsymbol{P}$ and let

$$
D=\bigoplus_{\aleph_{0}} R\left(P^{\infty}\right)
$$

Then $D$ is not supplemented.

Proof. Consider the submodule

$$
N=\bigoplus_{n \in N} R / P^{n}
$$

of $D$. Since $N$ is reduced $N / B$ is reduced for every bounded submodule $B$ of $N$. If $S \leqq D$ such that $D=N+S$ then $N /(N \cap S) \cong$ $D / S$ is divisible so that $N \cap S$ is not bounded. Because of Theorem 3.6, $N \cap S$ is not small in $N$. Hence, by $4.1, S$ is not a minimal supplement of $N$.

We are ready to determine the structure of torsion-free supplemented modules. A torsion-free module is called completely decomposable if it is a direct sum of submodules of rank one [5; p. 112].

Proposition 4.13. Let $M$ be a torsion-free module over the proper Dedekind domain $R$. If $M$ is supplemented then $M$ is completely decomposable of finite rank, and $M \neq 0$ implies $R$ local. 
Proof. Let $M=N \oplus D$ where $N$ is reduced and $D$ is divisible. By 4.5 and Lemma $4.12, D$ has finite rank and, by $4.11, N$ is small. By 3.7, if $R$ is local, $N$ is free of finite rank and $M$ has the desired structure. Assume $R$ is not local. By 2.4 the only torsionfree sum-irreducible $R$-module is the zero-module. Thus, $M$ cannot contain a maximal submodule since any minimal supplement of it would have to be sum-irreducible. It follows that $J(M)=M$, hence either $M=0$ or, using 4.5 again, $K$ is supplemented. To show that the latter is impossible, let $R \leqq A \leqq K$ such that $A / R=R\left(P^{\infty}\right)$ for some $P \in P$. By Theorem 3.6, $A$ is not small, and $\boldsymbol{P} \neq\{P\}$ implies $A \lessgtr K$. Thus, using 4.10, a minimal supplement of $A$ in $K$ would need to be a proper nonzero divisible submodule of $K$. The nonexistence of such a submodule completes the proof.

A module $A$ is called artinian if its lattice of submodules satisfies the descending chain condition $[1 ; \mathrm{p} .127]$. Clearly, artinian modules are supplemented. If $A=A_{P}$ for some $P \in \boldsymbol{P}$, then the following statements are equivalent: (1) $A$ is artinian; (2) $A$ has finite rank; (3) $A$ is a direct sum of finitely many submodules each of which is either isomorphic to $R\left(P^{\infty}\right)$ or to $R / P^{n}, n \geqq 0$ an integer [10; p. 49, Exercise 49].

Frequent use will be made of the fact that, for $R$ a Dedekind domain, a torsion $R$-module $T$ is supplemented if and only if, for each $\boldsymbol{P} \in \boldsymbol{P}, T_{P}$ is supplemented.

TheOREM 4.14. Let $M$ be a module over the proper Dedekind domain $R$. Then $M$ is supplemented if and only if $M=Y \bigoplus_{P \in P} M_{P}$ where, for each $P \in P, M_{P}$ is a direct sum of a bounded and an artinian submodule, and $Y \neq 0$ implies $R$ local and $Y$ completely decomposable torsion-free of finite rank.

Proof. Clearly, sum-irreducible modules are supplemented. Thus, if $M$ has the described structure, by 2.4 and $4.7, M$ is supplemented provided $\bigoplus_{P \in P} M_{P}$ is supplemented. Again, this will be the case if we can show that every $R$-module $B$ with $P^{n} B=0, P \in P$, $n$ a positive integer, is supplemented. But this follows from the fact that such $B$ can be regarded as module over the perfect ring $R / P^{n}[1 ;$ p. $315,28.4$ ]; by $[12 ;$ p. 528, Folgerung], $B$ is supplemented. Conversely, assume $M$ is supplemented and let $M=D \oplus N$ with $D$ divisible and $N$ reduced. By 4.7 and 4.11, $N$ is small and, because of 4.12 , each $D_{P}$ is artinian. Furthermore, 4.13 implies that $M$ is torsion or $R$ is local and that in a decomposition.

$$
D=\bigoplus_{P \in P} D_{P} \oplus \bigoplus_{1} K
$$

the index set $I$ must be finite. Applying Proposition 3.7 to the 
small module $N$ completes the proof.

Corollary 4.15. Let $R$ be a Dedekind domain and let $C$ be one of the following classes of $R$-modules:

(i) the class of all supplemented torsion $R$-modules;

(ii) the class of all reduced supplemented $R$-modules.

Let $\mathbf{0} \rightarrow L \rightarrow M \rightarrow N \rightarrow 0$ be an exact sequence of $R$-modules. Then $M \in C$ if and only if both $L \in C$ and $N \in C$. In particular, each module in $C$ is amply supplemented.

Proof. If all submodules of a module $M$ are supplemented then $M$ is amply supplemented. Thus, the last part of the proposition follows from the first. For $R$ local, submodules of free modules are free [10; p. 44, Lemma 15]. Apply Theorem 4.14.

Our "supplemented" modules and those of [7] are called "komplementiert" in $[8 ; 11 ; 12 ; 20]$; the "perfect" modules of [16] coincide with our "amply supplemented" modules. In these papers, various properties of the $R$-module $M$ were shown to be equivalent to $M$ being either supplemented or amply supplemented. These equivalences, though not restricting the ring $R$, required $M$ to meet stringent conditions, like being projective $[11 ; 12]$, quasi-projective $[7 ; 8 ; 12 ; 16]$, or $\sigma[M]$-projective $[20]$. We take the opposite approach: in order to clarify the relationships of the various properties for an arbitrary $R$-module $M$, we require $R$ to be a Dedekind domain but remove the restrictions placed on $M$.

In the following theorem, we still require $M$ to be reduced. The restriction to nonlocal domains in the second part of 4.17 cannot be dispensed with: if $p$ is a rational prime and $Z_{(p)}$ denotes the localization of $Z$ at $(p)$, then every quasi-projective $Z_{(p)}$-module is either free or a direct sum of pairwise isomorphic finite cyclic submodules [6; p. 7, Proof of Theorem]. Thus supplemented modules over arbitrary Dedekind domains need not have quasiprojective covers.

If certain projectivity conditions are imposed upon $M$, the word "weak" in (iii) and (iv) of 4.17 can be omitted (cf. [12; p. 526, SATZ, and 15; p. 24], [7; p. 179, 3.7], [20; p. 135, 2.6], [7; p. 178, $3.2]$, [8; p. 278 , SATZ 6], [16; p. $92,2.3]$ ). In order to show that in general, this is not possible we provide

EXAMPLe 4.16. Let $R=Z$, let $p \in Z$ be a positive prime, and let $M=\langle a\rangle \oplus\langle b\rangle$ where $a$ has order $p$ and $b$ has order $p^{2}$. Then $M$ is supplemented, $J(M)=p M$, but the direct decomposition 


$$
M / J(M)=\langle(a+b)+p M\rangle \oplus\langle b+p M\rangle
$$

cannot be lifted to a direct decomposition of $M$; also, the nonsmall submodule $\langle a+b\rangle$ contains no nonsmall (i.e. nonzero) direct summand of $M$.

THEOREM 4.17. The following properties of the reduced module $M$ over the proper Dedekind domain $R$ are equivalent.

(i) $M$ is supplemented.

(ii) $M$ is amply supplemented.

(iii) $J(M) \ll M, M / J(M)$ is semi-simple, and weak direct decompositions of $M / J(M)$ can be lifted to $M$.

(iv) Every nonsmall submodule of $M$ contains a nonsmall weak direct summand of $M$.

( $\mathrm{v}) \quad J(M) \ll M$ and $M$ is a direct sum of sum-irreducible submodules.

(vi) $\quad M=F \oplus \bigoplus_{P \in P} M_{P}$ where, for each $P \in P, M_{P}$ is bounded, and $F \neq 0$ implies $R$ local and $F$ free of finite rank.

If $R$ is not local then, in addition, (i)-(vi) are equivalent to each of the following:

(vii) $M$ has a quasi-projective cover which is supplemented.

(viii) Every M-generated module has a quasi-projective cover.

Proof. Reduced torsion-free modules of rank one over local domains are cyclic [10; p. 48]. Thus, by 4.14, (i) is equivalent to (vi) and, by 3.7 and 2.4, (vi) in turn is equivalent to (v). According to 4.9, (iii) implies (i) and, using 4.15, (i) implies (ii). Assume (ii). Then, because of $4.11, M$ is small, and the first two conditions in (iii) follow from 3.5 and 4.8. In order to verify the third one, put $J=J(M)$ and let $M / J=B_{1} / J \bigoplus_{W} B_{2} / J$. Then $M=B_{1}+B_{2}, B_{1} \cap$ $B_{2}=J$, and $M$ being amply supplemented implies $M=C_{1} \bigoplus_{W} C_{2}$ for some $C_{i} \leqq M$ such that $C_{i} \leqq B_{i}, i=1,2$. Clearly, $C_{i}+J=B_{i}, i=$ 1,2, and we have derived (iii) from (ii). Clearly (ii) implies (iv). Assume (iv). If $S$ is a weak direct summand contained in $J$ then, by $4.2, J(S)=S \cap J=S$, so that $S=0$ since $M$ is reduced. Thus, $J \ll M$ and, in view of 3.7 , (vi) holds if we can show that $M$ is torsion or $R$ is local. Suppose $M$ is not torsion. Then $M$ is generated by its nontorsion elements, and $M$ reduced implies $R x \geqq J$ for some $x \in M$ with $R x \cong R$. If $R$ is not local, $P x \notin J$ for some ideal $P \in \boldsymbol{P}$, thus $P x$ contains a nonsmall weak direct summand of $M$ which must be of the form $I x$ for some proper ideal $I \neq 0$ of $M$. This is impossible by Lemma 2.2 , proving $R$ is local. The first six conditions have been shown to be equivalent.

For the remainder of the proof, assume $R$ is not local. Then, 
by 3.10 , the equivalent properties (i) and (vi) together imply both (vii) and (viii). By 4.5, (i) follows from (vii). Assume (viii). Then, for each $P \in \boldsymbol{P}, R\left(P^{\infty}\right)$ is not $M$-generated, according to 3.9. Thus, $M$. must be torsion and each $M_{\cdot P}$ is bounded. We have derived (vi) from (viii) and the proof is completed.

For notational convenience, define, for any $R$-module $M$,

$$
M_{0}=M /\left[\bigoplus_{P \in \boldsymbol{P}} M_{P}\right] .
$$

The zero ideal of $R$ is prime; thus, by 4.14 and 2.4 , a divisible module $D$ is supplemented if and only if, for each prime ideal $Q$ of $R, D_{Q}$ is a direct sum of finitely many sum-irreducible submodules. By 4.7, the presence of a submodule $D$ of this form does not affect whether or not a module is supplemented.

This motivates the following definition. Recall that $d M$ denotes the maximal divisible submodule of $M$.

Definition 4.18. The submodule $J$ of $M$ is almost small in $M$ if $J / d J$ is small in $M / d J$ and, for each prime ideal $Q$ of $R,(d J)_{Q}$ is a direct sum of finitely many sum-irreducible submodules.

Weakening $J(M) \ll M$ to the postulate that $J(M)$ be almost small in $M$, some of the conditions of Theorem 4.17 remain equivalent for nonreduced modules. It is clear, in light of Lemma 3.9, that (vii) and (viii) cannot be retained. Conditions (ii) and (iv) do not carry over either: nonreduced supplemented modules need not be amply supplemented, nor do nonsmall submodules necessarily contain nonsmall weak direct summands. This can be seen from the following

EXAMPLE 4.19. Let $R$ be a local Dedekind domain with maximal ideal $P$ which is not complete in its $P$-adic topology, let $K$ be the quotient field of $R$, and let $M=K \oplus K$. By 4.14, $M$ is supplemented. Let $N$ be an indecomposable submodule of $M$ of rank two [10; p. 46, Theorem 19]. By $3.7, N$ is not small so that $M=N+T$ for some $T \supsetneqq M$. Since $M / T$ is divisible, any minimal supplement of $T$ in $M$ must be divisible, by 4.10 . Since $N$ is reduced, $N$ contains no minimal supplement of $T$ in $M$. Thus, $M$ is supplemented but not amply supplemented. For the same reason, the nonsmall submodule $N$ contains no nonsmall weak direct summand of $K$ since weak direct summands of divisible modules are divisible (cf. 4.2). Also, note that $N$ is not supplemented.

THEOREM 4.20. Let $M$. be a module over the proper Dedekind domain $R$. Then the following conditions are equivalent. 
(1) $M$ is supplemented.

(2) $J(M)$ is almost small in $M ; M / J(M)$ is semi-simple; and weak direct decompositions of $M / J(M)$ can be lifted to $M / d M$.

(3) $J(M)$ is almost small in $M$, and $M$ is a direct sum of sum-irreducible submodules.

(4) $\quad M=Y \oplus \bigoplus_{P \in P} M_{P}$ where each $M_{P}$ is a direct sum of an artinian and a bounded submodule, and $Y \neq 0$ implies $R$ local and $Y$ completely decomposable torsion-free of finite rank.

Proof. Let $M=N \oplus D$ where $D=d M$, and put $J=J(M)$. Then

$$
J=J(N) \oplus J(D)=J(N) \oplus D .
$$

Thus, $J$ is almost small in $M$ if and only if $J(N) \ll N$ and $D=d J$ is supplemented. Since $N$ is reduced, the theorem follows readily from 4.14 and 4.17 , observing that

$$
N / J(N) \cong M / J(M) \cong(M / d M) /(J(M) / d M)=(M / d M) / J(M / d M)
$$

$[1 ;$ p. $121,9.15]$.

\section{REFERENCES}

1. F. W. Anderson and K. R. Fuller, Rings and Categories of Modules, SpringerVerlag, New York, 1974.

2. H. Bass, Finitistic dimension and a homological generalization of semiprimary rings, Trans. Amer. Math. Soc., 95 (1960), 466-488.

3. S. Eilenberg, Homological dimension and syzygies, Ann. of Math., 64 (1956), 328336.

4. C. Faith, Algebra II Ring Theory, Springer-Verlag, Berlin, 1976.

5. L. Fuchs, Infinite Abelian Groups, Academic Press, New York, 1973.

6. L. Fuchs and K. M. Rangaswamy, Quasi-projective abelian groups, Bull. Soc. Math. France, 98 (1970), 5-8.

7. J. S. Golan, Quasiperfect modules, Quart. J. Math. Oxford (2), 22 (1971), 173-182.

8. G. Hauger, Aufsteigende Kettenbedingung fur zyklische Moduln und perfekte Endomorphismenringe, Acta Math. Acad. Sci. Hungar., 28 (1976), 275-278.

9. I. Kaplansky, Modules over Dedekind rings and valuation rings, Trans. Amer. Math. Soc. 72 (1952), 327-340.

10. - Infinite Abelian Groups, Revised Edition, The University of Michigan Press, Ann Arbor, 1969.

11. F. Kasch, Moduln und Ringe, B. G. Teubner, Stuttgart, 1977.

12. F. Kasch and E. A. Mares, Eine Kennzeichnung semi-perfekter Moduln, Nagoya Math. J., 27 (1966), 525-529.

13. M. D. Larsen and P. J. McCarthy, Multiplicative Theory of Ideals, Academic Press, New York, 1971.

14. W. W. Leonard, Small Modules, Proc. Amer. Math. Soc, 17 (1966), 527-531.

15. E. A. Mares, Semi-perfect modules, Math. Z., 82 (1963), 347-360.

16. Y. Miyashita, Quasi-projective modules, perfect modules, and a theorem for modular lattices, J. Fac. Sci. Hokkaido Univ., 19 (1966), 86-110.

17. B. Pareigis, Radikale und Kleine Moduln, Bayer. Akad. Wiss. Math.-Natur. Kl. 
S.-B. 2 (1966), 185-199.

18. K. M. Rangaswamy and N. Vanaja, Quasi projectives in abelian and module categories, Pacific J. Math., 43 (1972), 221-238.

19. D. W. Sharpe and P. Vamos, Injective Modules, Cambridge University Press, 1972.

20. R. Wisbauer, $\sigma$-semiperfekte und $\sigma$-perfelte Moduln, Math. Z., 162 (1978), 131-138.

21. L. E. Wu and J. P. Jans, On quasi projectives, Illinois J. Math., 11 (1967), 439-448.

Received September 6, 1980 and in revised form January 28, 1981.

University of Houston, Central Campus

HousTON, TX 77004 


\section{PACIFIC JOURNAL OF MATHEMATICS}

\section{EDITORS}

DONALD BABBITT (Managing Editor)

University of California

Los Angeles, CA 90024

Hugo RossI

University of Utah

Salt Lake City, UT 84112

C. C. Moore and Arthur Agus

University of California

Berkeley, CA 94720
J. DugundJI

Department of Mathematics

University of Southern California

Los Angeles, CA 90007

R. FinN and J. MiLgRAM

Stanford University

Stanford, CA 94305

\section{ASSOCIATE EDITORS}
R. ARENS
E. F. BECKENBACH
B. H. NeumanN
F. WOLF
K. YoSHIDA

\section{SUPPORTING INSTITUTIONS}

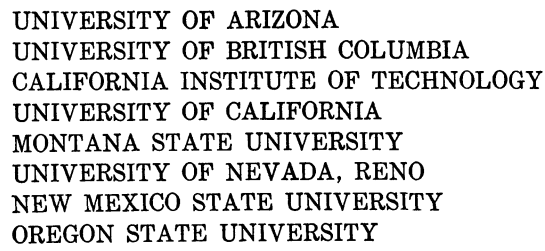

UNIVERSITY OF ARIZONA

UNIVERSITY OF BRITISH COLUMBIA CALIFORNIA INSTITUTE OF TECHNOLOGY UNIVERSITY OF CALIFORNIA MONTANA STATE UNIVERSITY

UNIVERSITY OF NEVADA, RENO NEW MEXICO STATE UNIVERSITY OREGON STATE UNIVERSITY

\author{
UNIVERSITY OF OREGON \\ UNIVERSITY OF SOUTHERN CALIFORNIA \\ STANFORD UNIVERSITY \\ UNIVERSITY OF AAWAII \\ UNIVERSITY OF TOKYO \\ UNIVERSITY OF UTAH \\ WASHINGTON STATE UNIVERSITY \\ UNIVERSITY OF WASHINGTON
}

The Supporting Institutions listed above contribute to the cost of publication of this Journal, but they are not owners or publishers and have no responsibility for its content or policies,

Mathematical parers intended for publication in the Pacific Journal of Mathematics should be in typed form or offset-reproduced, (not dittoed), double spaced with large margins. Please do not use built up fractions in the text of the manuscript. However, you may use them in the displayed equations. Underline Greek letters in red, German in green, and script in blue. The first paragraph or two must be capable of being used separately as a synopsis of the entire paper. Please propose a heading for the odd unmbered pages of less than 35 characters. Manuscripts, in triplicate, may be sent to any one of the editors. Please classify according to the scheme of Math. Reviews, Index to Vol. 39. Supply name and address of author to whom proofs should be sent. All other communications should be addressed to the managing editor, or Elaine Barth, University of California, Los Angeles, California, 90024 .

50 reprints to each author are provided free for each article, only if page charges have been substantially paid. Additional copies may be obtained at cost in multiples of 50 .

The Pacific Journal of Mathematics is issued monthly as of January 1966, Regular subscription rate: $\$ 114.00$ a year (6 Vol., 12 issues). Special rate: $\$ 57.00$ a year to individual members of supporting institution.

Subscriptions, orders for numbers issued in the last three calendar years, and changes of address shoud be sent to Pacific Journal of Mathematics, P.O. Box 969, Carmel Valley, CA 93924, U.S.A. Old back numbers obtainable from Kraus Periodicals Co., Route 100, Millwood, NY 10546.

\section{PUBLISHED BY PACIFIC JOURNAL OF MATHEMATICS, A NON-PROFIT CORPORATION}

Printed at Kokusai Bunken Insatsusha (International Academic Printing Co., Ltd.). 8-8, 3-chome, Takadanobaba, Shinjuku-ku, Tokyo 160, Japan.

Copyright (C) 1982 by Pacific Journal of Mathematics Manufactured and first issued in Japan 


\section{Pacific Journal of Mathematics}

Vol. 100, No. $2 \quad$ October, 1982

Kenneth F. Andersen, On the transformation of Fourier coefficients of

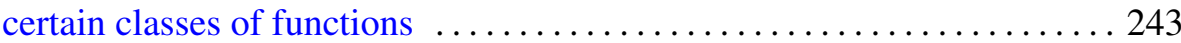

Steven Albert Bleiler, Realizing concordant polynomials with prime knots

Reinhard Bürger, Functions of translation type and solid Banach spaces of functions

Ulrich Daepp, The saturation of $k$-analytic rings and topological equivalence of associated analytic set germs .................. 271

Persi W. Diaconis and David Amiel Freedman, On the maximum difference between the empirical and expected histograms for sums . . . 287

David Amiel Freedman, On the maximum of scaled multinomial

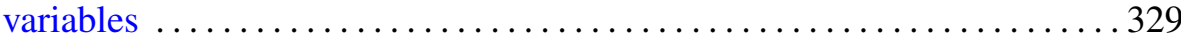

Persi W. Diaconis and David Amiel Freedman, On the difference between the empirical histogram and the normal curve, for sums. II ......... 359

Persi W. Diaconis and David Amiel Freedman, On the mode of an

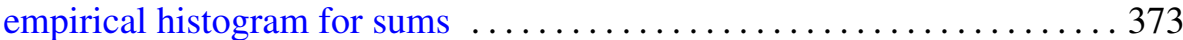

Jutta Hausen, Supplemented modules over Dedekind domains 387

Elyahu Katz, A moduli representation for the classification of twisted tensor

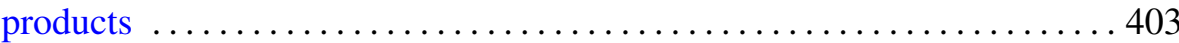

H. C. Madhekar and N. K. Thakare, Biorthogonal polynomials suggested by the Jacobi polynomials

Ted R. Pettis, Collections of covers of metric spaces 425

Ryōtarō Satō, Maximal functions for a semiflow in an infinite measure space

Michael Jay Stob, Invariance of properties under automorphisms of the lattice of recursively enumerable sets 